\title{
Poverty identification for a pro-poor health insurance scheme in Tanzania: reliability and multi-level stakeholder perceptions
}

\author{
August Kuwawenaruwa ${ }^{1 *}$, Jitihada Baraka ${ }^{1}$, Kate Ramsey ${ }^{3}$, Fatuma Manzi ${ }^{1}$, Ben Bellows ${ }^{4}$ and Josephine Borghi ${ }^{1,2}$
}

\begin{abstract}
Background: Many low income countries have policies to exempt the poor from user charges in public facilities. Reliably identifying the poor is a challenge when implementing such policies. In Tanzania, a scorecard system was established in 2011, within a programme providing free national health insurance fund (NHIF) cards, to identify poor pregnant women and their families, based on eight components. Using a series of reliability tests on a 2012 dataset of 2,621 households in two districts, this study compares household poverty levels using the scorecard, a wealth index, and monthly consumption expenditures.
\end{abstract}

Methods: We compared the distributions of the three wealth measures, and the consistency of household poverty classification using cross-tabulations and the Kappa statistic. We measured errors of inclusion and exclusion of the scorecard relative to the other methods. We also gathered perceptions of the scorecard criteria through qualitative interviews with stakeholders at multiple levels of the health system.

Findings: The distribution of the scorecard was less skewed than other wealth measures and not truncated, but demonstrated clumping. There was a higher level of agreement between the scorecard and the wealth index than consumption expenditure. The scorecard identified a similar number of poor households as the "basic needs" poverty line based on monthly consumption expenditure, with only $45 \%$ errors of inclusion. However, it failed to pick up half of those living below the "basic needs" poverty line as being poor. Stakeholders supported the inclusion of water sources, income, food security and disability measures but had reservations about other items on the scorecard.

Conclusion: In choosing poverty identification strategies for programmes seeking to enhance health equity it's necessary to balance between community acceptability, local relevance and the need for such a strategy. It is important to ensure the strategy is efficient and less costly than alternatives in order to effectively reduce health disparities.

Keywords: Poverty identification, Insurance, Wealth index, Consumption expenditure, Tanzania

\section{Background}

User fees gained widespread use as a means of alleviating pressure on constrained public health facility and district budgets in many sub-Saharan African countries and reducing unnecessary demand by populations $[1,2]$. Yet user fees represent a financial burden for the poor and other vulnerable groups, and may reduce demand

\footnotetext{
* Correspondence: ajoachim@ihi.or.tz

${ }^{1}$ Ifakara Health Institute, Plot 463, Kiko Avenue Mikocheni, P.O. Box 78 373, Dar es Salaam, Tanzania

Full list of author information is available at the end of the article
}

for health care services among these groups [3-5]. As a result, most countries have systems in place to exempt the poor from paying user fees in public facilities.

While it is relatively easy to identify patient groups (such as pregnant women), identifying the poor is more challenging. One commonly accepted method is to measure poverty through means testing $[6,7]$ based on income or consumption expenditure, with the latter being more reliable, less prone to seasonal variation and easier to collect in most rural settings $[6,8,9]$. However, in low income countries it can be difficult to measure 
individual income or consumption expenditure without undertaking costly surveys. The most common approaches to identify the poor for user fee exemptions include: proxy means testing, whereby a limited number of household or individual characteristics are used to judge poverty [10], the community or participatory wealth ranking method, whereby community members or leaders rank neighbouring households [11], and geographic targeting, whereby all residents in a given geographic area (e.g., village, district) are identified as poor (this is sometimes combined with community and proxy means testing) [12]. Geographical targeting is less precise relative to other methods used to target poor households, but is typically lower in cost and administratively simple $[13,14]$. A lack of reliable methods to identify poor people in the community has affected implementation of exemption policies in low and middle income countries [15].

Previous studies have examined how these approaches compare to each other in terms of identifying the poor $[16,17]$. One study considered the acceptability of community wealth ranking to identify the poor for a community health insurance scheme in Burkina Faso [18]. A study in Ghana compared means testing (based on consumption expenditure), proxy means testing (based on a wealth index) and the participatory wealth ranking approaches in terms of their classification of the poor [19]. Both proxy means testing and participatory ranking were found to have errors of inclusion, but participatory ranking was more acceptable to stakeholders. Another study compared these approaches in terms of their cost [19]. However, most existing studies considered these poverty measures hypothetically, rather than assessing actual measures being employed within exemption programmes. Understanding the reliability and acceptability of different approaches to poverty assessment is important to inform more effective exemption policies.

We compare the reliability of a proxy means testing approach being used to identify the poor within a pro-poor health insurance scheme in Tanzania (a scorecard - Table 1), relative to two conventional wealth measures (a wealth index and consumption expenditure), and the acceptability of the scorecard method among stakeholders at various levels in the country.

\section{Methods}

\section{Study setting}

User fees are charged at all public health facilities in Tanzania, but specific groups are exempt from their payment including: pregnant women, children under five years of age, people with chronic conditions, the elderly, and the poor. Households can enrol in the Community Health Fund (CHF), a community-based health insurance scheme, to avoid having to pay user fees at public primary care facilities, at an annual premium of between TZS 5,000 (US \$2.40) to TZS 30,000 (US \$ 14.40) depending on the district. The poor are also supposed to be eligible for free enrolment in the CHF. The National Health Insurance Fund (NHIF) also provides free access to services across all service levels and types of facility to its members. However, coverage is limited to the formal sector, and contributions are made directly at source (6\% of salary). National health insurance coverage is around $15 \%$ [20].

There are various guidelines and commonly accepted practices in the Tanzanian health sector for identifying the poor, with the result that at the local level there is significant discretion for community leaders and health workers to determine which, if any, measures are to be applied [21, 22]. According to Mubyazi this policy failure to define 'who are the poor' or how the poor should be assessed has caused confusion among healthcare providers [21]. Some of the people who are eligible for exemptions are still incurring out of pocket payments when seeking health care at public facilities [23], with variation across districts [4, 23].

In 2010 the Tanzania National Health Insurance Fund (NHIF), with technical support from GFA Consulting group and funds from the German Development Bank (KfW) introduced a programme to give poor pregnant women free NHIF health insurance cards in Mbeya and Tanga regions, and free CHF cards to their households. The scheme initially used individual targeting to identify poor women for enrolment in the scheme using a scorecard. It was expected that the scorecard system might subsequently be used for poverty identification elsewhere in the country. The scorecard is based on eight indicators (housing materials, roof type, sanitation, fuel for cooking, remoteness from health providers, income, food security (measured in terms of the number of meals per day) and the number of dependants and those with disabilities (Table 1). A score rate of between 8 and 16 points qualifies the person as eligible to receive the insurance card [24]. The poverty dimensions included on the scorecard were adapted from those used in voucher programmes elsewhere in SubSaharan Africa [25].

\section{Data collection methods}

To assess the reliability of the poverty scorecard, a survey of 2,623 households of women who had delivered within the previous 12 months was conducted in April 2012 across two districts (Mbarali and Kilolo) as part of an evaluation of the free health insurance card programme [26]. Households were sampled using a modified version of the Expanded Program on 
Table 1 Household Screening Form

\begin{tabular}{|c|c|c|}
\hline \multirow{2}{*}{$\begin{array}{l}0.1 \text { Is there a woman residing in the household who has given birth } \\
\text { during the previous } 12 \text { months? }\end{array}$} & Yes 01 & \multirow{2}{*}{$\begin{array}{l}\text { If no, terminate } \\
\text { interview }\end{array}$} \\
\hline & No 02 & \\
\hline \multirow{2}{*}{$\begin{array}{l}0.2 \text { Does this woman currently have any insurance cover or has she } \\
\text { had cover in the previous } 12 \text { months? }\end{array}$} & Yes 01 & \\
\hline & No 02 & \\
\hline \multirow[t]{7}{*}{0.3 Which kind of insurance does this woman have/did she have? } & 01 NHIF BIMA WAZAZI & \multirow{7}{*}{$\begin{array}{l}\text { Terminate interview if } \\
01,02,03,05,06,88\end{array}$} \\
\hline & 02 NHIF EMPLOYER CONTRIBUTION & \\
\hline & 03 CHF/TIKA BIMA WAZAZI & \\
\hline & $04 \mathrm{CHF} / \mathrm{TIKA}$ SELF CONTRIBUTION & \\
\hline & $05 \mathrm{SHIB}$ & \\
\hline & 06 PRIVATE INSURANCE & \\
\hline & 88 OTHER (SPECIFY) & \\
\hline
\end{tabular}

0.4 Interviewer instruction:

Please score households from 1 to 3 depending on the nature of their house and their responses to the following questions.

$0.4 a$ Nature of the house

0.4b Distance to nearest health facility walking

0.4c Water source

0.4d Fuel for cooking

0.4e Toilet facilities

$0.4 f$ Daily income

$0.4 \mathrm{~g}$ Average number of meals per day

$0.4 \mathrm{~h}$ Number of children/vulnerable children in household

0.4i Total score for household

ADD SCORES FROM 0.4a-0.4i
01 Mud house with thatched roof

02 Mud house with iron sheet roof

03 Brick house

0160 minutes or more

02 between 30 and 60 minutes

03 less than 30 minutes

01 River/stream/lake

02 Shared well or tap

03 Private well or tap

01 Firewood or animal dung or no facilities

02Charcoal

03 Kerosene, gas or electricity

01 Bush

02 Shared latrine

03 Private latrine or toilet

01 Less than 1500 Tsh per day

03 More than 3000 Tsh per day

011 meal per day

022 meals per day

033 meals per day

01 More than 4 children or one of your children is disabled or suffers from a chronic disease.

023 or 4 children

03 Less than 3 children

01 Score 8-13= poor

02 Score 14-18= average

03 Score 19-24 = rich
02 Between 1500-3000 Tsh per day

Score |
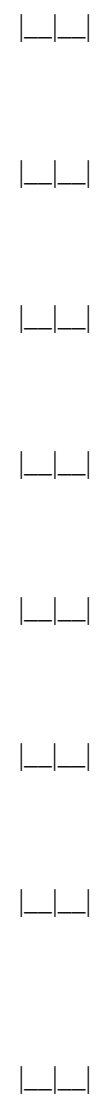

NB: Each component had three options, with a score ranging from one to three per an answer. Aggregation of the score made a total of 8 to 24 based on the score assigned on the observed/responded answer. Household scoring 8 - 16 qualified for the programme. In additional to the 8 questions households were asked about asset ownership, utilities and housing characteristics and total consumption expenditure in a typical month

Name of household head......

Name of woman who delivered in previous 12 months.

If household is eligible and consent is obtained, continue with interview: 
Immunization (EPI) type sampling scheme from villages surrounding 48 health facilities within the districts [26]. Before the tool was administered, the household was screened using the NHIF poverty scorecard. The aim was to sample households of differing wealth status: two-thirds scoring poor or average and the remaining one-third non-poor and ineligible for the insurance card. Households were then asked a series of questions on ownership of assets and housing particulars and average monthly consumption expenditure in relation to food, clothing, housing services (rent), energy, transport, communication and spending on health (e.g., medicine, transport for health care seeking and consultation fees).

Purposive sampling was used to select 39 key informants for interviews across district, health facility and community levels. At the health facility level, three facilities were chosen to represent at least one facility at each level of the health system and based on geographic location in the district and annual caseload of deliveries. National poverty criteria used by the Tanzanian Social Action Fund (TASAF) were then reviewed with district managers to identify three of the poorest villages and corresponding hamlets in the catchment of each facility. Eight focus groups were conducted in these communities with three among men and six among women who had a child under the age of one year. The participants were asked about their impressions of the pro-poor health insurance program, including the criteria for identifying the poor. Interviews and focus groups were conducted by Tanzanian researchers in Swahili and digitally recorded. Interviewers used recordings and field notes to produce full transcripts which were translated into English. One of the researchers, who is fluent in Swahili and English, conducted quality checks on the transcripts and translations.

\section{Data analysis methods}

We created a wealth index including indicators relating to housing characteristics (water source, toilet type, source of fuel used for cooking, source of light, nature of flooring, nature of roof, nature of walls, number of rooms) and assets (electricity, radio, TV, DVD player, mobile phone, iron, fridge, sewing machine, tables, sofas, cupboards, motorbike, car, bank account) using polychoric principal component analysis (PCA). Polychoric PCA is preferred since the standard PCA assumes a linear relationship between the variables [8] which does not apply well to dummy or categorical variables [27]. Further it has been argued that transforming categorical variables into dummy variable for use in PCA may lead to loss of potential information especially when the categories have a meaningful ordering [27]. Total monthly consumption expenditure was estimated as the sum of expenditure across the seven expenditure components identified above, in Tanzanian Shillings. When using the NHIF scorecard we estimated the overall score for each household.

We examined the distribution and probability density functions of each of these three measures (wealth index, consumption expenditure and NHIF scorecard) graphically to assess the degree of skewness, clumping and truncation by plotting histograms. Truncation can arise when there is little difference in measured values among households at the extreme of the distribution (e.g., poor and very poor). If an insufficient number of asset indicators are used, then households will be grouped (clumped) together in a small number of groups, which is also problematic for the generation of wealth groupings (e.g., terciles). To assess agreement of classification of households according to the three different measures, we derived terciles based on the wealth index, consumption expenditure and the NHIF score, and estimated Kappa statistics to assess the degree of agreement of classification between the three measures. The Kappa statistic is a measure of agreement between categorical variables, which takes into account the agreement expected by chance $[28,29]$, and a value of less than 0.5 is taken to indicate poor agreement between measures.

We also examined errors of exclusion and inclusion of households when using the NHIF scorecard as compared to the wealth index and consumption expenditure. Exclusion error is defined as the number of the poor households excluded over the total number of poor households. Whereas inclusion error is defined as the number of non-poor households identified as poor over the total number of households [30, 31]. When using a proxy means testing approach to poverty identification, an agreed threshold is needed to establish who is poor and who isn't. When using consumption expenditure or income to measure wealth, the basic needs poverty line can be used to identify poor households, with all households falling below this line being considered as poor. The World Bank research routinely uses \$1.25 per person per day as a benchmark to identify the poor below that figure [32]. When using a wealth index based on asset ownership information, for example, a relative wealth ranking is typically carried out, whereby those within the lowest quintile or tercile are considered to be poor. In principle, households could be classified according to the national distribution by drawing on Demographic and Health Survey data for example. In this study, we use the following approaches to determine who is poor for each wealth measure.

When considering household monthly consumption expenditure, we used the National Bureau of Statistics definition of basic needs poverty as consumption expenditure 
below 36,482 TZS per adult equivalent per month [33]. To estimate the total number of adult equivalents per household we used the following formula [34].

The equivalence scale used is

$$
\mathrm{e}_{h}=\left(\mathrm{A}_{h}+0.5 \mathrm{~K}_{h}\right)^{0.7}
$$

Where: $\mathrm{A}_{h}$ is the number of adults (above 15 years) in household $h$ and $\mathrm{K}_{h}$ the number of children (0-15 years) in the household.

When considering the wealth index, we assumed that those in the lowest tercile were poor. Finally, when considering the NHIF scorecard, we assumed that those scoring in the range of $8-16$ were poor, as this was the criterion for determining eligibility for the scheme.

After qualitative data collection, the researchers read all of the English transcripts for familiarisation. Thematic analysis was conducted by two researchers based on the poverty identification criteria. The two researchers each conducted coding of the interviews independently using NVIVO 9.0 (QSR International). Variation in coding between the two was discussed until consensus achieved. Responses across the different categories of respondent were compared using framework analysis.

\section{Results}

\section{Household survey}

Just under $90 \%$ of the surveyed households were headed by males. The majority of household heads $(87.6 \%)$ were Christian (Table 2). Almost $80 \%$ of the surveyed household heads had completed primary education, most $(85.7 \%)$ of the households were subsistence farmers, and only $2.9 \%$ were enrolled in the CHF insurance scheme.

\section{Distribution of wealth measures}

The histograms based on the wealth index and consumption expenditure are skewed to the left, indicating that the majority of the sample are of lower wealth levels, with a more limited number of observations from higher wealth groups (Figs. 1 and 2). Both distributions also suffer from leftward truncation. In contrast, the histogram based on the scorecard was relatively normally distributed (Fig. 3). However, clumping was an issue for the scorecard, which may be explained by the smaller number of indicators used in its generation.

There was greater consistency in the overall classification of households by wealth category when comparing the wealth index and the NHIF scorecard (61\% classified in the same tercile), than consumption expenditure ( $43 \%$ classified in the same tercile)
Table 2 Households Demographic Characteristics

\begin{tabular}{|c|c|c|}
\hline Variable & All $(N=2,623)$ & \\
\hline $\begin{array}{l}\text { Characteristics of the Household } \\
\text { head }\end{array}$ & $n$ & $\%$ \\
\hline Married & 912 & 34.8 \\
\hline Gender is male & 2,297 & 87.6 \\
\hline \multicolumn{3}{|l|}{ Religion } \\
\hline Catholic & 871 & 33.2 \\
\hline Other Christians & 1,427 & 54.4 \\
\hline Muslim & 251 & 9.57 \\
\hline Other & 74 & 2.8 \\
\hline \multicolumn{3}{|l|}{ Education } \\
\hline No school & 264 & 10.1 \\
\hline Some primary education & 189 & 7.2 \\
\hline $\begin{array}{l}\text { Completed primary education/ } \\
\text { Some secondary education }\end{array}$ & 2,054 & 78.3 \\
\hline $\begin{array}{l}\text { Secondary education and } \\
\text { above }\end{array}$ & 114 & 4.4 \\
\hline \multicolumn{3}{|l|}{ Occupation } \\
\hline Formal employment & 82 & 3.1 \\
\hline Not working & 102 & 3.9 \\
\hline Subsistence farmer & 2,247 & 85.7 \\
\hline Self employed/small business & 189 & 7.2 \\
\hline \multirow[t]{2}{*}{ Insured by the CHF } & 2,623 & 2.9 \\
\hline & $n$ & Mean [s.d] \\
\hline $\begin{array}{l}\text { Number of children under } 5 \text { in the } \\
\text { household }\end{array}$ & 2,623 & $2.85[1.70]$ \\
\hline $\begin{array}{l}\text { Number of elderly over } 65 \text { in the } \\
\text { household }\end{array}$ & 2,623 & $0.05[0.24]$ \\
\hline Household size & 2,623 & $4.89[1.64]$ \\
\hline Average age of household head & 2,569 & $36.76[11.64]$ \\
\hline \multicolumn{3}{|l|}{ Wealth measures } \\
\hline NHIF Scorecard - Index value & 2,623 & $15.99[2.23]$ \\
\hline $\begin{array}{l}\text { Monthly consumption } \\
\text { expenditure in TZS }\end{array}$ & 2,623 & $\begin{array}{l}98,070.74 \\
{[85551.55]}\end{array}$ \\
\hline Wealth index & 2,623 & $-2.58 \mathrm{e}^{-09}[2.55]$ \\
\hline
\end{tabular}

(Table 3). The Kappa statistic was also higher indicating greater reliability between the wealth index and the scorecard, although in both cases the Kappa statistic remained below 0.50 .

The basic needs poverty line identified the greatest number of poor households at just under $60 \%$ (Table 4). A similar proportion of households were identified as poor by the scorecard, but a much lower level (33.36 \%) were identified as poor by the wealth index (i.e., in the lowest tercile). Additional analysis showed that less than $33.36 \%$ of our surveyed respondents fall into the lowest tercile in relation to the national Demographic and Health survey when comparing their wealth index 


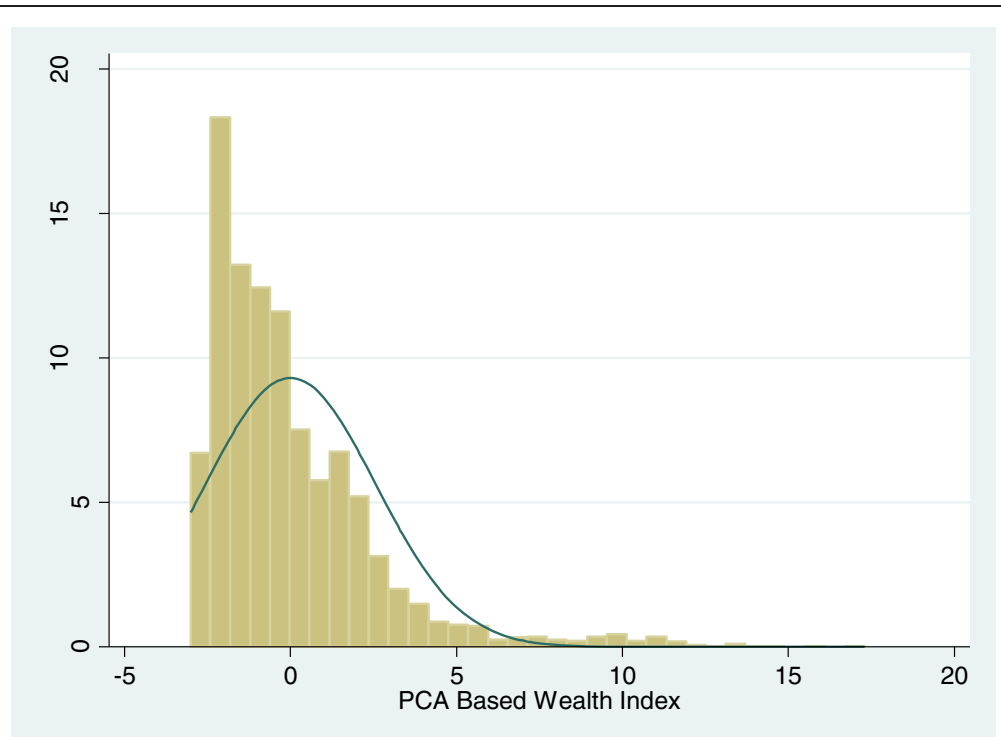

Fig. 1 Wealth index derived using PCA. Distribution of the household wealth index scores created using principal component analysis (PCA) extraction method in Mbarali and Kilolo District: Tanzania

values. About $68 \%$ of the poor (basic needs poverty) were identified by the scorecard, compared to over $91 \%$ of those in the bottom tercile of the wealth index. The scorecard resulted in errors of exclusion of around $32 \%$ and $9 \%$ with respect to the basic needs poverty line and the lowest wealth tercile respectively; while the error of inclusion was $45 \%$ with respect to the basic needs poverty line, and $42 \%$ with respect to the lowest wealth tercile.

\section{Key informant interviews and FGDs}

Among the key informants, $48 \%$ were female and $52 \%$ male. For government key informants (including health providers), total years of service in the government ranged from 4 to 30 years with a range of 2 to 12 years in their current position. Respondents from nongovernmental organizations had been in their current roles approximately 4 to 5 years. The age of women participating in the focus groups ranged from 22 to 45 years old, with a similar range among men (24 to 46 years old).

There was a notably high level of consensus among stakeholders across different levels regarding the poverty criteria. Although positive about the overall aims of the program, respondents exhibited scepticism about the

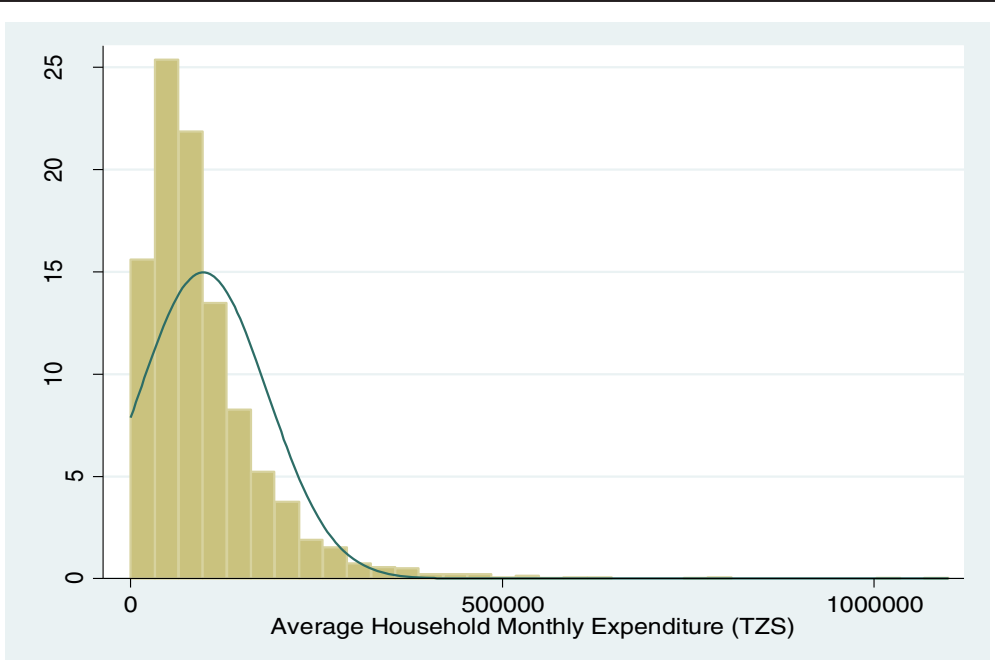

Fig. 2 Reported average monthly consumption expenditure (TZS). Distribution of the household based on the reported consumption expenditure during the household survey in Mbarali and Kilolo District: Tanzania 


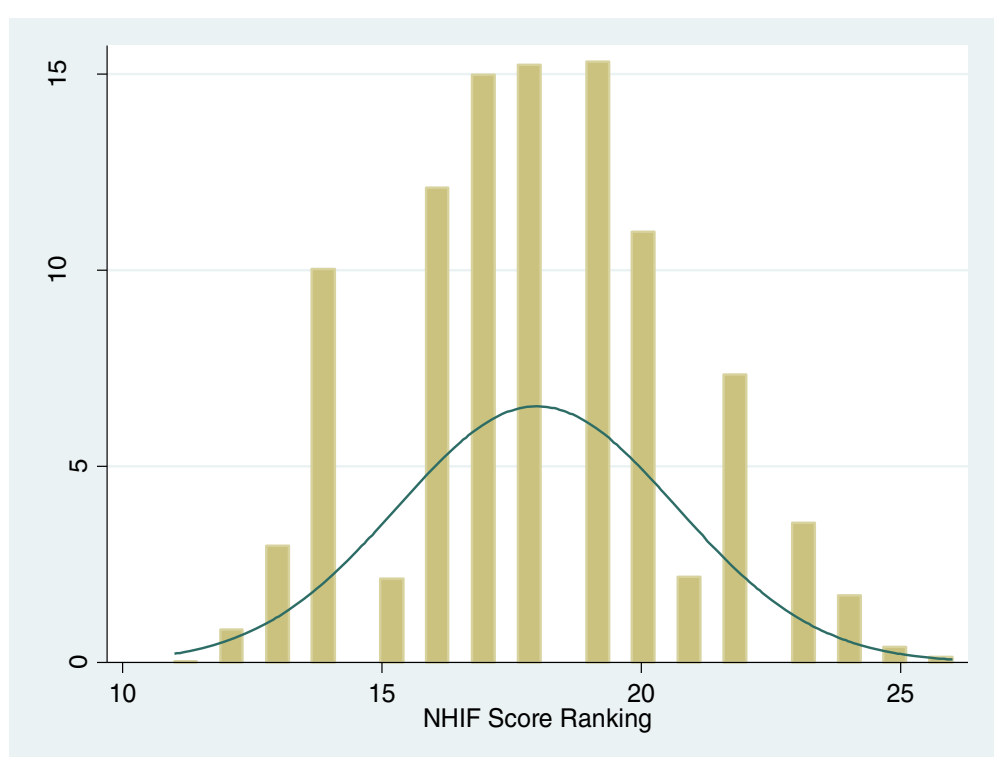

Fig. 3 National Health Insurance Fund (NHIF) Scorecard. Distribution of the household based on the NHIF scorecard within the surveyed households in Mbarali and Kilolo District: Tanzania

poverty targeting criteria in the scorecard and emphasized the general conditions of poverty throughout their context. Nonetheless, there was general consensus that water sources, daily income, and average number of meals per day were good criteria for identifying the poor in their context.

".....For a pregnant woman to eat one meal is not right. She is supposed to eat more for the health of the mother and growth of the baby, but when she eats one meal it means she has nothing to eat. She thinks, 'if I eat three times today, what about tomorrow?"'

(Male FGD participant)

"... Women walk long distances to fetch shallow water in the rivers and streams despite some of the villages having community tap water. But still, women spend a lot of time to get water" (Health provider)

"....Money is everything. If the woman is selling doughnuts and she gets very little profit ..... and she is craving meat then she cannot even afford to buy it." (Female FGD participant)

Participants described various reasons behind their perceptions of the score card criteria. For the criteria related to housing materials, respondents indicated that this may be appropriate but could also reflect individual or cultural preferences rather than poverty. Given a certain amount of resources, they felt that people will make different decisions that might not align with these criteria. Others, such as the presence of a toilet, were considered a poor indicator of poverty and instead an indicator of knowledge regarding sanitation and hygiene.

".... One can have a nice house with iron sheets, but they do not have food to eat, maybe they inherited the house from their forefathers. Others they have mud and thatch houses but they have plenty of cattle and more food in storage." (Female FGD participant)

"...some of the families do not think having a better house is a priority. They give priority to other things like education. Others live in a thatched house and when you look, their standards of living are better - eating well, dressing well and doing all things well - but they do not take all the children to school."(Community leader)

"..... Regarding the toilet, it's not a criterion for poverty at all, it's all about health of the people and ignorance of the people. For the toilet even if you are poor, [you] cannot fail to dig a pit latrine,"

(Male FGD participant)

When discussing the indicator distance to a health facility, many of the respondents felt that this was not useful for individual level targeting due to the fact that decisions about where to build facilities are determined by government actors. In addition, in some settings, there are poor women living very close to the facilities. 
Table 3 Movement of households between terciles when comparing NHIF scorecard results to the wealth index and consumption expenditure

\begin{tabular}{lcc}
\hline $\begin{array}{l}\text { Movement of Households } \\
\text { (PCA) }\end{array}$ & $\begin{array}{l}\text { Wealth index } \\
\text { Expenditure }\end{array}$ & $\begin{array}{l}\text { Consumption } \\
\text { Proportion of households ranked in } \\
\text { the same tercile }\end{array}$ \\
$\begin{array}{l}\text { Proportion of households moving } \\
\text { by one tercile }\end{array}$ & 32.18 & 42.64 \\
$\begin{array}{l}\text { Proportion of households moving } \\
\text { by two terciles }\end{array}$ & 6.83 & 34.93 \\
Kappa statistic & $0.373^{* * *}$ & $0.122^{* * *}$ \\
\hline
\end{tabular}

Statistically significant are ${ }^{*} p<0.05{ }^{* *} p<0.01{ }^{* * *} p<0.001$

"I think, she can be living far or near and still be poor." (Health provider)

"...but we should not say she is poor on her own. It is national poverty because they could build health facility every 30 kilometres as the policy says that there should be health facility in each village"

(District respondent)

There were divergent opinions about whether the number of children should be considered in determining a woman's poverty status which did not appear to follow patterns based on type of respondent.

Table 4 Identification of the poor and errors of inclusion and exclusion

\begin{tabular}{|c|c|c|}
\hline \multirow[b]{2}{*}{ Identification Mechanism } & \multicolumn{2}{|c|}{ All $(n=2,623)$} \\
\hline & $n$ & $\%$ \\
\hline Wealth index (PCA) (bottom tertile) & 875 & 33.36 \\
\hline $\begin{array}{l}\text { Consumption Expenditure (CE) } \\
\text { (below "basic needs" poverty line) }\end{array}$ & 1,540 & 58.71 \\
\hline Scorecard (grade between 8-16) & 1,534 & 58.48 \\
\hline $\begin{array}{l}\text { Among those below the national } \\
\text { basic needs poverty line }\end{array}$ & \multicolumn{2}{|c|}{$(n=1540)$} \\
\hline Identified as poor by the scorecard & 1,049 & 68.12 \\
\hline Identified as poor by the wealth index & 643 & 41.75 \\
\hline Among those in the lowest wealth tercile & \multicolumn{2}{|c|}{$(n=875)$} \\
\hline Identified as poor by the scorecard & 795 & 90.86 \\
\hline \multicolumn{3}{|l|}{ In relation to the basic needs poverty line } \\
\hline $\begin{array}{l}\text { Errors of inclusion (\% of those above the } \\
\text { basic needs poverty classified as poor by scorecard) }\end{array}$ & 485 & 44.78 \\
\hline $\begin{array}{l}\text { Errors of exclusion (\% of those below basic } \\
\text { needs poverty not classified as poor by scorecard) }\end{array}$ & 491 & 31.88 \\
\hline \multicolumn{3}{|l|}{ In relation to the wealth index } \\
\hline $\begin{array}{l}\text { Errors of inclusion (\% classified as poor by the } \\
\text { scorecard who are not in lowest tercile) }\end{array}$ & 739 & 42.27 \\
\hline $\begin{array}{l}\text { Errors of exclusion (\% of those in lowest tercile not } \\
\text { classified as poor by scorecard) }\end{array}$ & 80 & 9.14 \\
\hline
\end{tabular}

Some respondents recognized that additional children might put a strain on available resources, while others noted that some families might have many children and yet be in a good position financially. Responses regarding children with disabilities followed a similar pattern. For both, respondents indicated that it would need to be considered in light of other factors.

\section{“...It can be true or not true, because someone can have many children - more than six - but have a good environment. Another one might have two children or one, but fail to take care [of] the family..." (Influential Female Community Member)}

"If you have many children, you have to spend a lot so expenditures are high. So that can cause the family to be poor and fail even to pay for the health services." (District manager)

"It will depend, because what are the causes of the disability? The experts they can say that maybe the disability was caused by lacking basic things...But disability as disability cannot cause poverty. For those who pray they say that is just God's wish, he has seen the creation should be this way.

(Community leader)

"[having a child with a disability] also can contribute, because you can have additional burdens. You can even fail to do other tasks because you are caring for this child." (Health Provider)

When asked about additional criteria to consider, a number of respondents mentioned widows and young women citing that they will have less access to resources because of their status. They noted that some criteria such as clothing were important indicators likely to result in discrimination at the health facility. Whether children are attending school was also mentioned by a number of respondents.

"If a teacher or the wife of one of the civil servants because she dresses properly and you go to the dispensary and you are pregnant, it is discouraging because we stay a very long time waiting for the services, whereas they just call her and leave you behind."

(Female FGD participant)

"Even though not all those who don't go to school are poor, for primary education, every parent would like his or her child to at least be able to write and read. She or he may fail to take his or her child to secondary school, but not primary education. (District manager) 
Issues of how many cows they have, what land they have for farming, and whether they have money in the bank were also raised as potential indicators of poverty.

When asked their views about how to identify the poor, FGD respondents tended to say that the local government representatives had the ability to identify the poor. Yet, a number of respondents also expressed a lack of confidence in village leaders to fairly identify the poor. Some indicated concern about bias from the village leaders. One FGD respondent described how this had recently occurred in a maize distribution program for the poor. Another described how the reliability of these leaders varies across villages - with some providing aid when needed and others routinely avoiding work.

\section{Discussion}

The identification and targeting of the poor in relation to health programmes is one approach being used by policy makers to promote equity in health coverage. However, for such an approach to be effective in reaching its goal of enhancing health equity, it relies on the accurate assessment of poverty and identification of the poor. While there have been numerous studies comparing different approaches to poverty measurement, there have been few evaluating mechanisms used to identify the poor in health programmes, with some exceptions [18]. This paper set out to compare the poverty scorecard method used to identify households eligible for a free health insurance card to enhance maternal health care access, relative to two conventional methods of individual wealth ranking, a wealth index and monthly consumption expenditure, and to assess the acceptability of the scorecard method to key stakeholders.

The scorecard technique, when used to rank households, produced a distribution that was less skewed than other wealth measures and not truncated. However, the distribution demonstrated more clumping than the other measures of wealth, which may reduce its ability to differentiate between poor and very poor households. There was greater consistency in the overall classification of households by wealth between the wealth index and the scorecard method, than consumption expenditure, although the Kappa statistic was lower than 0.5 in both cases.

The scorecard identified a similar number of poor households to the basic needs poverty line defined in terms of a threshold of monthly consumption expenditure per adult equivalent, with only $45 \%$ being errors of inclusion. However, it failed to pick up a third of those living below the basic needs poverty line as being poor, meaning that a large proportion of the poor would not have been considered eligible for the free health insurance programme.
Figures were similar when comparing the scorecard to a poverty threshold determined by the wealth index (the bottom wealth tercile), although errors of inclusion were higher, as fewer individuals were classified as poor when using the wealth index threshold. We found a much lower level of errors of inclusion, and similar levels of errors of exclusion to that reported elsewhere [30]. This demonstrates the ability of proxy means testing to yield better results in different settings, a finding also illustrated previously [30], especially when the wealth proxies are locally determined and deemed relevant. Indeed, the degree of agreement between different approaches to poverty identification has been found to vary by context [30]. However, we were unable to examine the effect of context in this study as our study sites were fairly homogenous and rural.

Among stakeholders interviewed, there was general consensus that water sources, daily income, and average number of meals per day were good criteria for identifying the poor in their context. Food security, asset ownership, and disability have also been identified as important determinants of poverty in participatory wealth ranking exercises elsewhere (e.g., $[18,19,35])$. However, stakeholders expressed scepticism about the ability of other indicators to reliably identify the poor (sanitation, housing materials, roof type, number of children, and distance from the health facility), indicating that these might be the result of individual choices or structural factors beyond their control.

Household characteristics identified as important in other studies were not included in the scorecard, such as social exclusion [18, 19, 35], age [18], appearance [19], land and livestock ownership, and being childless [35]; however, these criteria, other than appearance, were not mentioned by stakeholders interviewed in this study as being relevant.

\section{Conclusion}

When choosing poverty identification strategies for health programmes aimed at enhancing equity, community acceptability and local relevance must be balanced against the need for a reliable and efficient strategy that can be employed easily and at minimum cost [36]. A proxy means testing approach (a scorecard) used to identify poor households for free health insurance can be effective in identifying the poor, increasing the affordability of health care among this group, but consideration of the implementation process surrounding a given poverty identification strategy should also be examined, as this may vary by context and individual targeting may be open to misuse. Further examination of this issue would be an important area for future research. 


\begin{abstract}
Abbreviations
CE: Consumption expenditure; CHF: Community Health Fund; FGD: Focus Group Discussion; KfW: German Development Bank; NHIF: National Health Insurance Fund; PCA: Principal component analysis; TZS: Tanzanian shilling (US $\$ 1==2084$ TZS).
\end{abstract}

\section{Competing interests}

The authors declare that they have no competing interests.

\section{Authors' contributions}

AK, participated in data collection, analysis, interpretation of data and drafted the manuscript; Baraka J, participated in data collection and analysis; KR developed the study protocol, participated in qualitative data analysis and critically reviewed the manuscript and approved final version; FM, developed the study protocol and critically reviewed the manuscript and approved final version. BB, critically reviewed the study protocol and objectives, edited the manuscript and approved the final version. Borghi J, developed the study protocol, guided the quantitative analysis, participated in the interpretation of data, drafted the manuscript and approved the final version. All authors read and approved the final manuscript.

\section{Authors' information}

AK, is a Research Scientist has been involved in health financing, impact evaluation and costing of health care interventions at the Ifakara Health Institute. AK has a BA and an MA in Economics.

JBaraka, is a Research Officer, qualitative expert at the Ifakara Health Institute. $\mathrm{JB}$ has a BA in sociology and currently is undertaking Masters of Public Health at The Muhimbili University of Health and Allied Sciences (MUHAS) K.R., is a Senior Research Officer at Columbia University, Mailman School of Public Health, New York, NY USA, and has been a technical advisor to the Ifakara Health Institute for the past 6 years. KR has an MPH from Columbia University and is currently a Doctor of Public Health student there as well. FM, is a senior health economist at Ifakara Health Institute, she leads the health systems thematic group. FM has worked on the evaluation of several health system projects at Ifakara, and has a PhD in Epidemiology and Public Health.

B.B, is an Associate with the Population Council's Reproductive Health program in Lusaka, Zambia. Bellows has MPH in Epidemiology/Biostatistics and Social Behaviour and PhD in Epidemiology

J.Borghi, is a Senior Lecturer at the London School of Hygiene \& Tropical Medicine, and has been working as technical advisor to the Ifakara Health Institute since 2007. JB has a BA, an MSc in Health Economics and a PhD in Health Economics.

\section{Acknowledgements}

We would like to acknowledge the assistance of the District Medical Officers, CHF coordinators, NHIF coordinators and the facility-in-charges from the sampled facilities in each of the districts for their support during the study. We also acknowledge the assistance of the following NHIF Headquarter, Mbeya and Iringa Zone Offices and other members of the management team. Thanks also to Population Council (via a grant from the Bill and Melinda Gates Foundation) for funding the study and to the European Commission who supported the time of two of the researchers involved in the study as part of the UNITAS project.

\section{Author details}

'Ifakara Health Institute, Plot 463, Kiko Avenue Mikocheni, P.O. Box 78 373, Dar es Salaam, Tanzania. ${ }^{2}$ Department of Global Health and Development, London School of Hygiene \& Tropical Medicine, London, UK. ${ }^{3}$ Columbia University, Mailman School of Public Health, New York, NY, USA. ${ }^{4}$ Population Council, Lusaka, Zambia.

Received: 16 April 2015 Accepted: 17 November 2015 Published online: 01 December 2015

\section{References}

1. Chuma J, Okungu V. Viewing the Kenyan health system through an equity lens: implications for universal coverage. Int J Equity Health. 2011;10:22.

2. Sharma S, Smith S, Sonneveldt E, Pine M, Dayaratna V, Sanders R. Formal and Informal Fees for Maternal Health Care Services In Five Countries POLICY Working Paper Series No. 16, 2005(16).
3. Hercot D, Meessen B, Ridde V, Gilson L. Removing user fees in the health sector: a review of policy processes in six sub-Saharan African countries. Health Policy Plan. 2011;26(Suppl 2):ii16-29.

4. Maluka SO. Why are pro-poor exemption policies in Tanzania better implemented in some districts than in others? Int J Equity Health. 2013;12(1):80.

5. Lagarde M, Palmer N. The impact of user fees on access to health services in low- and middle-income countries. Cochrane Database Syst Rev. 2011:4:CD009094.

6. Filmer D, Pritchett LH. Estimating wealth effects without expenditure data-or tears: an application to educational enrollments in states of India. Demography. 2001;38(1):115-32.

7. Vyas S, Kumaranayake L. Constructing socio-economic status indices: how to use principal components analysis. Health Policy Plan. 2006;21(6):459-68

8. Moser, C. and A. Felton. The Construction of an Asset Index Measuring Asset Accumulation in Ecuador Caroline Moser and Andrew Felton CPRC Working Paper 87 ISBN 1-904049-86-9. CPRC WOrking Paper 87, 2007(July).

9. Coady P, Grosh M, Hoddinott J. The Targeting of Transfers in Developing Countries: Review of Experience and Lessons. Washington DC: The World Bank; 2003.

10. Coady D, Parker S. Program Participation under Means-Testing and Self-Selection Targeting Methods, in Discussion Paper 191. Washington: IFRl; 2005.

11. Collins B. An understanding of poverty from those who are poor. SAGE Publications. 2009;3:9-31.

12. Elbersa C, Fujii T, Lanjouwd P, Özlerd B, and Yine W. Poverty alleviation through geographic targeting: how much does disaggregation help? Journal of Development Economics. 2007;83:198-213.

13. Karlan D, Thuysbaert B. Targeting ultra-poor households in Honduras and Peru. 2013.

14. Domelen JV. Reaching the Poor and Vulnerable: Targeting Strategies for Social Funds and other Community-Driven Programs. 2007(0711).

15. Idd A, Yohana O, Maluka SO. Implementation of pro-poor exemption policy in Tanzania: policy versus reality. Int J Health Plann Manage. 2013;28(4):e298-309.

16. Alatas V, Banerjee A, Hanna R, Olken B, Tobias J. Targeting the Poor: Evidence from a Field Experiment in Indonesia. Am Econ Rev. 2012;102(4):1206-40.

17. Handa S, Huang C, Hypher N, Teixeira C, Soares FV, Davis B. Targeting effectiveness of Social Cash Transfer Programs in Three Africa Countries. J Dev Effect. 2012;4(1):78-108.

18. Souares A, Savadogo G, Dong H, Parmar D, Sié A, Sauerborn R. Using community wealth ranking to identify the poor for subsidies: a case study of community-based health insurance in Nouna, Burkina Faso. Health Soc Care Community. 2010;18(4):363-8.

19. Aryeetey G, Jehu-Appiah C, Kotoh AM, Spaan E, Arhinful DK, Baltussen R, et al. Community concepts of poverty: an application to premium exemptions in Ghana's National Health Insurance Scheme. Global Health. 2013;9:12.

20. Mtei G, Makawia S, Masanja H. Monitoring and evaluating progress towards Universal Health Coverage in Tanzania. PLoS Med. 2014;11(9), e1001698.

21. Mubyazi G. Practice as compared with other developing countries: Evidence from Godfrey Martin Mubyazi. East African Journal of Public Health. 2004;1(1):1-10

22. Burns M, Mantel M. Tanzania Review of Exemptions and Waivers. Dar es Salaam Tanzania: Ministry of Health and Social Welfare; 2006.

23. Kruk ME, Mbaruku G, Rockers PC, Galea S. User fee exemptions are not enough: out-of-pocket payments for 'free' delivery services in rural Tanzania. Trop Med Int Health. 2008;13(12):1442-51.

24. Pörksen J. Developing a participatory poverty grading tool. London: Marie Stopes Internationa; 2004.

25. Broch V. and A. Team. Improved Access for the Poor Pregnant Women to Improve Maternal Health and HIV-related Services in Tanzania Draft Interim Design Report 1 June-30 October 2010 T AB L E O F C O N T E N T S Targeting of Poor Pregnant Women. 2010(2007): p. 1-97.

26. Borghi J, Ramsey K, Kuwawenaruwa A, Baraka J, Patouillard E, Bellows B, et al. Protocol for the evaluation of a free health insurance card scheme for poor pregnant women in Mbeya region in Tanzania: a controlled-before and after study. BMC Health Serv Res. 2015;15:258.

27. Kolenikov S, Angeles G. Socioeconomic status measurement with discrete proxy variables: is principal component analysis a reliable answer? Review of Income and Wealth. 2009;55(1):128-65. 
28. Howe LD, Hargreaves JR, Huttly SR. Issues in the construction of wealth indices for the measurement of socio-economic position in low-income countries. Emerg Themes Epidemiol. 2008;5:3.

29. Carletta J. Assessing agreement on classification tasks: the kappa statistic. Comput Linguist. 1996;22(2):249-54

30. Aryeetey GC, Jehu-Appiah C, Spaan E, D'Exelle B, Agyepong I, Baltussen R. Identification of poor households for premium exemptions in Ghana's National Health Insurance Scheme: empirical analysis of three strategies. Tropical Medicine \& International Health. 2010;15(12):1544-52.

31. Coady DP, S.P. Program Participation under Means-Testing and SelfSelection Targeting Methods, in FCND Discussion Paper 2005: Washington.

32. World Bank. World Development Indicators. Washington, DC: World Bank; 2014

33. NBS, Household Budget Survey Main Report. 2011/2012, National Bureau of Statistics Dar es Salaam, Tanzania

34. Donnell O, Doorslaer E, Rannan-Eliya R, Somanathan A, Adhikari S, Harbianto $D$, et al. Who benefits from public spending on health care in Asia? In: Working Paper \#3. 2005.

35. Kanchebe Derbile E, van der Geest S. Repackaging exemptions under National Health Insurance in Ghana: how can access to care for the poor be improved? Health Policy Plan. 2013;28(6):586-95.

36. Domelen JV. Reaching the Poor and Vulnerable:Targeting Strategies for Social Funds and other Community-Driven Programs. 2007, World Bank.

\section{Submit your next manuscript to BioMed Central} and we will help you at every step:

- We accept pre-submission inquiries

- Our selector tool helps you to find the most relevant journal

- We provide round the clock customer support

- Convenient online submission

- Thorough peer review

- Inclusion in PubMed and all major indexing services

- Maximum visibility for your research 